\title{
Spatial Analysis of the Distribution of Reported Dengue Incidence in the National Capital Region, Philippines
}

\author{
Mark Anthony P. Pangilinan, Derice Paolo G. Gonzales, Robert Neil F. Leong and Frumencio F. Co \\ De La Salle University - Manila
}

\begin{abstract}
Background and Objective. With an aim of developing an effective disease monitoring and surveillance of dengue fever, this study intends to analyze the spatial distribution of dengue incidences in the National Capital Region (NCR), across four years of reported dengue cases.

Materials and Methods. Data used was provided by the Department of Health ( $\mathrm{DOH}$ ) consisting of all reported dengue cases in NCR from 2010-2013. For mapping and visualization, a shapefile of NCR was made readily available by www.philgis.org. Both Moran's I and Kulldorff's spatial scan statistics (SaTScan) were used to identify clusters across the same time period.

Results and Conclusion. The analyses identified significant clustering of dengue incidence and revealed that the northern cities of NCR, such as Caloocan, Malabon, Navotas and Valenzuela, exhibited high spatial autocorrelation using local Moran's I and Kulldorff's SaTScan. A temporal analysis of the results also suggested movement in increased dengue incidence through time, from the northwest cities to the northeast cities. Presence of spatial autocorrelation in dengue incidence suggests possible enhancements of early detection schemes for dengue surveillance. Moreover, the results of these analyses will be of interest to both policymakers and health experts in providing a basis for which they can properly allocate resources for the prevention and treatment of dengue fever.
\end{abstract}

Key Words: dengue fever, dengue surveillance, disease clustering, Moran's I, Kulldorff's spatial scan statistic

\section{Introduction}

Dengue is a vector-borne infection prevalent in many tropical and sub-tropical countries around the world. The infection can come from one of four very similar serotypes of the dengue virus, which are commonly carried by the Aedes aegypti mosquito. The Aedes aegypti mosquito is the

Partial results presented and won Second Place at the 15th Faculty-Student National Conference on Statistical Science: Unifying Diversity in Scientific Research, Student Paper Category, November 2014, University of the Philippines - Diliman, Quezon City, Philippines.

Corresponding author: Robert Neil F. Leong

Mathematics Department

De La Salle University

2401 Taft Avenue, Manila 1004 Philippines

Telephone: +6325360270

Email: robert.leong.04@gmail.com primary vector of dengue fever. Infection begins when a mosquito carrying the dengue virus bites a healthy individual. The virus can also be transmitted to potential vectors when healthy mosquitoes feed on infected humans. Dengue is observed to spread faster in tropical places such as countries in South East Asia. ${ }^{1}$ Some of the symptoms of mild dengue include high fever, rashes, fatigue, joint and muscle aches, nausea, and vomiting. Severe levels of dengue (i.e., dengue hemorrhagic fever and dengue shock syndrome) show much more serious symptoms such as nose bleeding, internal bleeding, and sudden hypotension. ${ }^{2}$ Severe levels of dengue can cause death when left untreated, making it imperative to study its epidemiology. The World Health Organization (WHO) estimates that there are 50-100 million recorded cases of dengue fever annually. In the Philippines, there were 150,864 recorded cases of infection from January to September in the year 2013, according to the Department of Health's (DOH) National Epidemiology Center (NEC). ${ }^{3}$

Currently, there are on-going initiatives, both nationwide and worldwide, that aim to increase dengue fever awareness, surveillance, and vector management. According to $\mathrm{DOH}^{4}$, the National Dengue Prevention and Control Program (NDPCP), which was implemented nationwide in 1998, specifies that observing proper surveillance of dengue infection in the country is a stepping stone to reducing the morbidity and mortality of epidemics. Also, the NDPCP aimed to increase the awareness of dengue fever to $100 \%$ nationwide, and to lessen incidences from 32 cases down to 20 cases per population of 100,000. Additionally, the Global Strategy for Dengue Prevention and Control, spearheaded by WHO, highlighted the importance of identifying environmental factors related to dengue incidence and recurring trends in the distribution of infection incidence over a geographical space. ${ }^{5}$ An analysis of the distribution patterns and clusters of dengue incidence may be used in further studies and programs regarding vector management and risk factors of dengue fever.

In response to the magnitude of dengue fever's prevalence and the urgency to study its epidemiology in detail, this study aims to use a spatial analysis in exploring the distribution of the incidence of dengue fever in the National Capital Region (NCR) of the Philippines by identifying statistically significant clusters of dengue incidence in the NCR, across years of reported dengue 
incidence data. Clustering occurs when regions in a study area exhibit similar values as its neighboring regions. Both global and local indicators of spatial autocorrelation, and Kulldorff's spatial scan (SaTScan) statistics will be computed and used for verification and identification of clusters in the regions under study. Additionally, several years of gathered data will be used for spatial analysis in order to study and analyze the temporal attributes of dengue incidence.

\section{Review of Related Literature}

\section{The Dynamics of Dengue Infection}

Dengue incidence has increased unimaginably within the last few decades. In a study by the WHO (2012), it ranked as the leading mosquito transmitted disease worldwide. Only nine countries experienced high levels of dengue incidence during the 1970s, but now, dengue is prevalent in more than 100 countries around the world. Over $40 \%$ of the world's population, approximately 2.5 billion people, are at risk of getting exposed to dengue fever. ${ }^{6}$ Most of these cases can be found in tropical and sub-tropical countries where approximately 3.3 billion people reside. ${ }^{7}$ Due to this fact, not only the people living in the tropics are at risk of getting the infection, but also the tourists or travelers, who can contract the disease when visiting these places. Because of this, it is possible that the dengue virus will spread to other countries. This, in turn, makes dengue even harder to eradicate and adds to the worldwide burden of dengue that many countries are currently facing. Based on a study conducted in South East Asia, it was estimated that there is a loss of 420 DALYs (disability-adjusted life year) per million per year, which is the same as that of meningitis, twice that of hepatitis, and one third that of HIV/AIDS. From an economic perspective, it is estimated that the cost of dengue fever or dengue hemorrhagic fever per case is USD12.39, which is greater than the cost per case of treating malaria. ${ }^{8}$

A high population density is important in the transmission of the virus, which makes people living in urban areas more susceptible to the disease. In 2008, majority of the world's population resided in urban areas. ${ }^{9}$ Unplanned urbanization causes a rapid increase in the range of disease vectors because substandard housing, inadequate water supply, solid waste, and sewage systems provide more larval habitats for the mosquitoes. ${ }^{5}$ It is in this context that the NCR proved to be a relevant region to be selected as the area to be used in this study.

\section{On-Going Anti-Dengue Initiatives}

The Western Pacific Region's branch of WHO currently has a surveillance program in implementation that regularly monitors the incidence of dengue and other diseases over two-week periods. It is a program under the management of the Emerging Disease Surveillance and Response unit of the WHO. This project is in line with WHO's global strategy for dengue prevention and control, which states that improved disease surveillance is very important in reducing the morbidity of the disease. ${ }^{5}$

Local anti-dengue initiatives include the NDPCP. Since its nationwide implementation in 1998, the NDPCP has conducted several awareness campaigns and vector management programs aimed to lessen incidence and mortality of dengue fever. ${ }^{10}$ While disease awareness and vector management are vital in dengue prevention and control, there seems to be a shortage of local surveillance and monitoring programs. This may be due to the initiative's focus on the eradication of vector breeding habitats and the education of the public regarding the dangers and risks of dengue fever. One program that caters to vector management is the Flood Control Bayanihan Zone Alliance, which was implemented in 2011. One of this program's objectives is to implement anti-flood measures, which in turn may lessen potential habitats for mosquitoes to use for breeding. ${ }^{11}$ The National Epidemiology Center regularly reports on dengue incidence and its distributions across age, time (in months), sex, and geographical space. However, the data only provides disaggregate statistics of the disease incidence as distributed over the aforementioned stratifications.

\section{Spatial Analysis}

Because not only geography plays a significant role in the transmission of dengue but also with the advancements of geographical information systems (GIS) data, spatial analysis must be appropriate as an initial step of analyzing dengue distribution patterns. Spatial analyses have many practical applications. One of the more noble uses of spatial analyses is in the field of epidemiology, or the study of incidence and distribution of diseases. Several researches that used spatial analyses to map the incidence of vectorborne diseases have already been conducted in some countries, such as Ecuador and the United States. A study in the United States used local Moran's I and Kulldorff's spatial scan (SaTScan) statistics to identify clusters of West Nile virus incidence at the county level across the country. This research identified large consistent clusters of the West Nile virus, as well as some movement over time in the virus incidence across counties. ${ }^{12}$ Another study conducted in Ecuador used spatial analysis to study the geographical distribution of dengue in the city of Guayaquil. Spatial data analysis indicated the presence of high spatial autocorrelation, and preliminary results hinted a higher probability of becoming infected in a place that already has infected people, in spite of the fact that dengue is not a contagious disease. ${ }^{13}$ It is these studies that motivated the adaptation of spatial autocorrelation methods to analyze dengue distribution in the local setting. 


\section{Materials and Methods}

\section{Data Preparation}

The National Capital Region of the Philippines, located at $14.56^{\circ} \mathrm{N}, 121.03^{\circ} \mathrm{E}$, consists of 16 cities and one municipality. It is the most densely populated region of the country and the region that reported the highest number of dengue cases in 2012. ${ }^{14}$ The NCR has a land area of approximately $636 \mathrm{~km}^{2}$ and experiences temperatures ranging on average from $22^{\circ} \mathrm{C}$ to $34^{\circ} \mathrm{C}$. Because the Philippine archipelago has a tropical climate, mosquitoes are able to thrive in most parts of the country.

Data used for the study was provided by the Regional Epidemiology and Surveillance Unit (RESU) of the DOH. The data presents all dengue cases (in daily frequencies) in the NCR that were reported to medical units under the DOH surveillance system from 2010 to 2013, with over 140,000 reported cases in the NCR over the four years. Fields included in each reported dengue case include the exact date of the case report, the medical unit that the case was reported to, the age and sex of the patient, and the city or municipality of the patient's residence.

The shapefile of the Philippines was made readily available by www.philgis.org (Figure 1). The variable used for Moran's I analyses is the quarterly dengue incidence rate expressed per 10,000 individuals of the population of the cities to eliminate the effect of varying susceptible population sizes. Meanwhile, the regional count of dengue incidence was used for the Kulldorff's spatial scan (SaTScan) statistics analyses. In the process of generating the results for the spatial analyses, QGIS was used to generate the centroids for the cities.

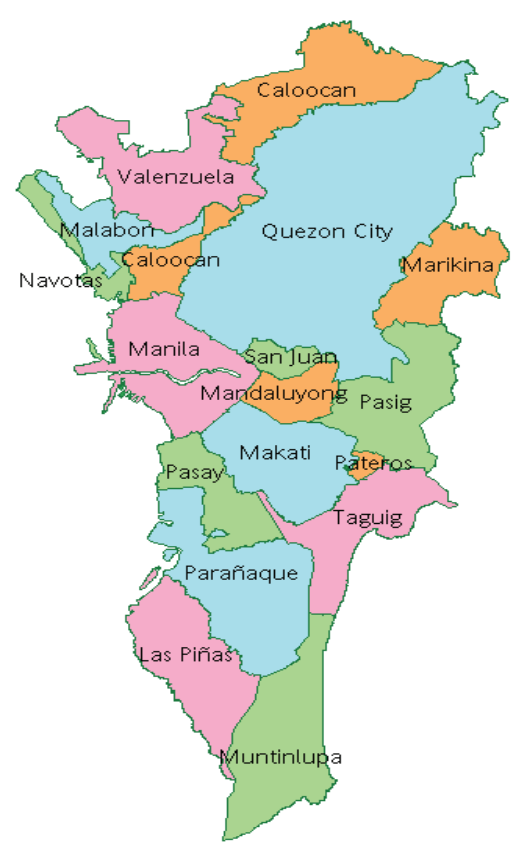

Figure 1. A Map of NCR with City Labels.

\section{Data Analysis}

After obtaining the raw data from $\mathrm{DOH}$, the data was then cleaned and prepared for analysis. Then, a binary weight matrix $W$ was generated by establishing a threshold for the distances between region centroids. Distance-based weights were used due to the large variations in size between the regions. It was observed that some regions may be close in proximity to each other but not necessarily contiguous. In summary,

$$
w_{i j}=\left\{\begin{array}{l}
1, \text { if } d_{i j} \leq q \\
0, \text { if } d_{i j}>q
\end{array}\right.
$$

where $d_{i j}$ is the distance between the centroids of regions $i$ and $j, q$ is the minimum distance for which each region has at least one neighbor. The $w_{i j}$ served as neighborhoodindicator, wherein two cities are considered to be neighbors if $w_{i j}$ is 1 , and non-neighbors otherwise. These $w_{i j}$ 's allow the spatial pattern assessment of dengue cases at the city-level.

After acquiring the required data fields needed for analysis, Moran's $I^{15}$ was then computed for each quarter across four years, using GeoDa $a^{\mathrm{TM}}$. Values of Moran's I were tested for significance with 99,999 permutations under a Monte Carlo procedure. Moran's I is a global index of spatial autocorrelation. The index is computed as follows:

$$
I=\left(\frac{1}{s^{2}}\right) \frac{\sum_{i=1}^{N} \sum_{i=1}^{N} w_{i j}\left(y_{i}-\bar{y}\right)\left(y_{j}-\bar{y}\right)}{\sum_{i=1}^{N} \sum_{i=1}^{N} w_{i j}},
$$

where $N=17$ is the total number of spatial units (i.e., cities) considered, $y_{i}$ is the dengue incidence rate at spatial unit $i, \bar{y}$ is the overall dengue incidence rate over the entire NCR, and $s^{2}$ is the variance of the dengue incidence rates across the different spatial units, which is computed as follows:

$$
s^{2}=\frac{1}{N} \sum_{i=1}^{N}\left(Y_{i}-\bar{Y}\right)^{2}
$$

After observing that statistically significant clustering was present in the study area for some of the quarters, local Moran's $I^{16}$ was then used to identify which cities or municipalities exhibited localized clustering in each quarter, which allow to pinpoint the individual clusters among the regions of the study area. The Monte Carlo permutation test was also used to test the significance of the local Moran's I values. For each spatial unit $i$, local Moran's $I$ is computed as:

$$
I_{i}=\frac{\left(y_{i}-\bar{y}\right)}{s} \sum_{j=1}^{N} w_{i j} \frac{y_{j}-\bar{y}}{s}
$$

where the same aforementioned definitions apply for $y_{i}, w_{i j}$, and $s$.

In addition to the local Moran's $I$ method used in identifying individual clusters in the study area, Kulldorff's spatial scan (SaTScan) statistics were also used to identify clusters of varying sizes across the same time period. Kulldorff's spatial scan statistic is a method of identifying 
specific clusters of varying sizes within a study area. This class of statistics uses zones with varying distance-based radii to compare the rates between clusters of regions. The statistic aims to generate circles consisting of regions whose incidence rates are significantly higher than the regions outside of the circles. A Poisson-based model was used for this study because the data consists of regional counts converted into incidence rates (per 10,000). In this model, it is desired to test the hypothesis that the probability $\pi_{\text {in }}$ of contracting the disease inside the circle is equal to the probability $\pi_{\text {out }}$ of contracting the disease outside the circle $\left(\pi_{\text {in }}=\pi_{\text {out }}\right)$. The alternative hypothesis is that the probability $\pi_{i n}$ inside the circle is significantly higher than the probability $\pi_{\text {out }}$ outside the circle $\left(\pi_{\text {in }}>\pi_{\text {out }}\right)$. The test statistic $D$, which is the ratio of the likelihood under the alternative hypothesis to the likelihood under the null hypothesis, can be simplified into the following equation:

$$
D(S)=\left\{\begin{array}{c}
\frac{\left(\frac{n_{Z}}{\mu(Z)}\right)^{n_{Z}}\left(\frac{n_{G}-n_{Z}}{\mu(G)-\mu(z)}\right)^{n_{G}-n_{Z}}}{\left(\frac{n_{G}}{\mu(G)}\right)^{n_{G}}}, \operatorname{if}\left(\pi_{\text {in }}>\pi_{\text {out }}\right), \\
1, \text { otherwise }
\end{array}\right.
$$

where $n_{z}$ is the number of cases inside the zone, $\mu(Z)$ is the population count inside the zone, $n_{G}$ is the number of cases outside the zone, and $\mu(G)$ is the population count outside the zone. ${ }^{17}$ Recorded results were generated and tested for significance through 99,999 permutations of the given data. The complete lists of these results were not presented in this article due to space restrictions but are available from the authors upon request.

\section{Results and Discussion}

\section{Global Autocorrelation}

Located in Table 1 are the global Moran's $I$ values for NCR per quarter. Seven out of 16 quarters returned statistically significant Moran's I values based on a 5\% level of significance. This denotes the presence of significant spatial autocorrelation in the study area for these quarters. Hence, existence of clusters during these seven periods is present. Significant values of the global Moran's I ranged from 0.1901 to 0.5244 . However, one quarter that was deemed not statistically significant returned a Moran's I value equal to 0.2672 , which is included in the interval.

Table 1. Aggregated Quarterly Incidence Rate and Moran's $I$ of Dengue in NCR

\begin{tabular}{ccccc}
\hline Year & Quarter & $\begin{array}{c}\text { Incidence Rate } \\
\text { (cases/10,000) }\end{array}$ & Moran's I & p-value \\
\hline \multirow{6}{*}{2010} & Q1 & 1.9037 & 0.3166 & $0.0480^{a}$ \\
& Q2 & 0.8257 & 0.1901 & $0.0125^{a}$ \\
& Q3 & 11.1125 & 0.5241 & $0.0045^{a}$ \\
& Q4 & 8.8192 & 0.0989 & 0.2286 \\
& Q1 & 5.3821 & 0.2672 & $0.0773^{b}$ \\
& Q2 & 2.1694 & 0.5244 & $0.0067^{a}$ \\
& Q3 & 10.7693 & 0.0219 & 0.3336 \\
& Q4 & 5.0835 & 0.1876 & 0.1242 \\
& Q1 & 4.7191 & 0.0233 & 0.3313 \\
& Q2 & 4.5656 & 0.0685 & 0.2652 \\
& Q3 & 13.5518 & -0.0614 & 0.4725 \\
& Q4 & 6.9847 & 0.4237 & $0.0191^{a}$ \\
& Q1 & 2.5717 & 0.1138 & 0.2112 \\
& Q2 & 2.2436 & 0.3248 & $0.0312^{a}$ \\
& Q3 & 9.1220 & 0.1108 & 0.2169 \\
& Q4 & 5.6773 & 0.4977 & $0.0087^{a}$ \\
\hline
\end{tabular}

${ }^{a}$ significant at $5 \%$ level of significance, ${ }^{\mathrm{b}}$ significant at $10 \%$ level of significance

Figure 2 shows a time series plot visualizing the seasonal pattern in the incidence rates from Table 1. Summarized in Table 2 are the quarterly rates per city from 2010 to 2013. It can be observed that the rates peak every third quarter of the year. This may be due to the rainy season in NCR during this time, which may propagate vector breeding in the study area. Using the F-test for the presence of seasonality, a significant seasonality was also found to be present at the 0.01 level of significance. The incidence rates found in Table 2 can be visualized through quintile maps as seen in Figures 3 and 4.

Table 2. Quarterly Dengue Incidence Rates (Cases/10,000) by City

\begin{tabular}{|c|c|c|c|c|c|c|c|c|c|c|c|c|c|c|c|c|}
\hline & \multicolumn{4}{|c|}{2010} & \multicolumn{4}{|c|}{2011} & \multicolumn{4}{|c|}{2012} & \multicolumn{4}{|c|}{2013} \\
\hline & Q1 & Q2 & Q3 & Q4 & Q1 & Q2 & Q3 & Q4 & Q1 & Q2 & Q3 & Q4 & Q1 & Q2 & Q3 & Q4 \\
\hline Caloocan & 1.4237 & 0.5843 & 11.5846 & 8.7976 & 5.1845 & 2.5788 & 8.8916 & 3.4855 & 3.8414 & 4.8622 & 11.4570 & 4.6271 & 2.4109 & 2.6259 & 7.9111 & 3.3243 \\
\hline Las Pinas & 1.1220 & 0.4343 & 5.8454 & 5.2844 & 4.3976 & 0.8506 & 4.1081 & 4.4700 & 3.1851 & 2.0631 & 8.4876 & 5.9359 & 1.5925 & 0.9411 & 7.0398 & 7.9627 \\
\hline Makati & 1.6445 & 0.6049 & 3.4213 & 4.7445 & 2.2494 & 0.9262 & 6.2377 & 4.4231 & 4.2152 & 2.0792 & 10.3395 & 5.9920 & 1.6634 & 1.0018 & 5.2926 & 5.1225 \\
\hline Malabon & 2.9717 & 1.4717 & 21.9054 & 18.2545 & 8.2641 & 4.1320 & 14.0942 & 6.9622 & 5.3207 & 6.3679 & 14.6036 & 8.7169 & 3.4245 & 2.3773 & 9.1697 & 5.8867 \\
\hline Mandaluyong & 4.0158 & 0.9431 & 9.0356 & 5.9325 & 4.7156 & 1.0040 & 6.6018 & 4.3809 & 3.8637 & 1.1865 & 14.9073 & 9.2790 & 2.5860 & 1.4299 & 6.7539 & 3.8029 \\
\hline Manila & 2.4634 & 0.7445 & 8.7521 & 10.9553 & 4.4668 & 1.6887 & 11.1308 & 9.0669 & 5.4716 & 4.4608 & 13.4490 & 9.8598 & 2.3121 & 1.6645 & 6.3734 & 7.3540 \\
\hline Marikina & 2.2869 & 1.3203 & 11.0574 & 4.4795 & 2.6170 & 1.3674 & 7.1909 & 1.7447 & 2.6642 & 3.1121 & 12.3541 & 4.1023 & 2.5698 & 1.9804 & 10.3501 & 4.4088 \\
\hline Muntinlupa & 0.6088 & 0.3044 & 7.8053 & & & & & & & 2.0003 & & & 1.7611 & 1.0001 & & 6.4573 \\
\hline Navotas & 4.2548 & 5.1379 & 18.3438 & 14.4502 & 8.9913 & 3.6527 & 10.2356 & 3.7731 & 2.7696 & 2.4887 & 5.2583 & 4.3351 & 2.1675 & 0.7627 & 4.8167 & 3.5724 \\
\hline Paranaque & 1.7003 & 0.4761 & 11.0521 & 10.3039 & 6.8693 & 1.1052 & 7.1583 & 6.5292 & 6.5972 & 4.3868 & 22.3592 & 16.8501 & 2.4825 & 1.3773 & 11.2391 & 10.9160 \\
\hline Pasay & 2.1636 & 0.7636 & 9.9779 & 6.8980 & 4.4290 & 1.4000 & 8.9597 & 5.9562 & 4.8108 & 3.5890 & 11.4542 & 13.5923 & 3.7163 & 2.2908 & 9.9015 & 9.8506 \\
\hline Pasig & 1.7618 & 0.3285 & 7.9729 & 9.5704 & 8.2715 & 1.1646 & 6.6888 & 4.3000 & 6.3454 & 4.7329 & 16.5131 & 10.2273 & 2.6875 & 1.6423 & 9.2270 & 7.5399 \\
\hline Pateros & 2.0266 & 0.7795 & 13.0949 & 18.7070 & 7.4828 & 2.0266 & 10.6007 & 5.9239 & 10.1330 & 2.4943 & 12.7831 & 6.0798 & 2.0266 & 1.2471 & 4.8327 & 2.6502 \\
\hline Quezon City & 1.6982 & 0.9342 & 14.2918 & 8.3571 & 5.5835 & 3.3349 & 17.4746 & 4.5805 & 5.3228 & 6.8544 & 15.6135 & 3.7875 & 3.5666 & 4.1134 & 13.6835 & 4.7507 \\
\hline San Juan & 2.0588 & 0.6588 & 14.1645 & 20.1762 & 7.4940 & 3.2117 & 12.0234 & 7.0823 & 7.5764 & 8.6470 & 27.3409 & 13.7528 & 2.9647 & 2.3882 & 12.1057 & 11.0352 \\
\hline Taguig & 1.1637 & 0.3569 & 5.6480 & 4.4067 & 5.4153 & 0.7913 & 6.6101 & 3.4292 & 3.9257 & 2.3740 & 9.8840 & 4.9032 & 0.9465 & 0.9000 & 7.1997 & 3.0878 \\
\hline Valenzuela & 2.3638 & 1.0081 & 17.3979 & 12.5140 & 7.6474 & 3.4240 & 11.9057 & 5.0404 & 4.5189 & 5.1273 & 12.8268 & 4.7970 & 1.9988 & 1.6859 & 6.7263 & 3.6152 \\
\hline
\end{tabular}




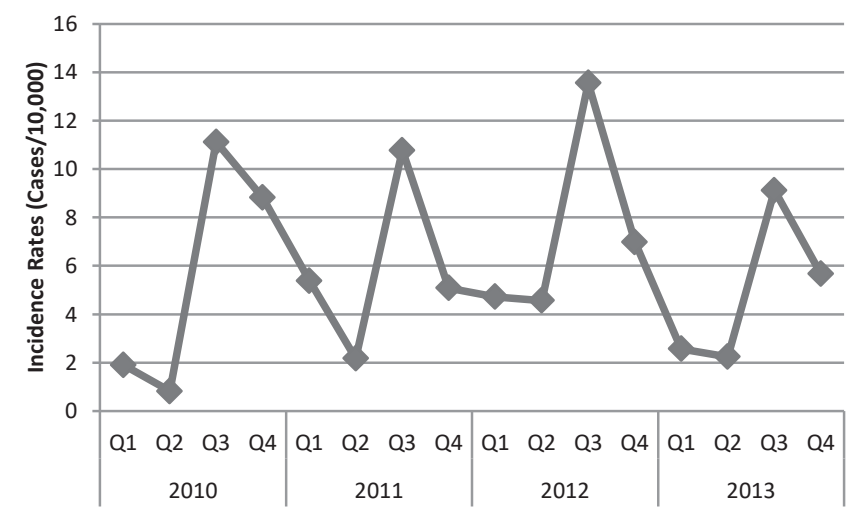

Figure 2. Dengue Incidence Rates (Cases/10,000) by Quarter.

\section{Localized Clusters}

Figures 5 and 6 display the SaTScan-generated results overlain on the local Moran's I cluster maps. Figure 5 reveals that the cities in the northern region exhibited high-high clustering, meaning that high values of incidence rates tend to occur close to each other. For the second quarter of the year 2011, the cities of Navotas, Malabon, Valenzuela, and Caloocan were identified as high-high clusters. Further observations on the local cluster maps also suggest that dengue incidence seems to move across the study area over time. It is observable that from the first quarter of 2010 to the second quarter of 2011, it appears as though high dengue incidence started from the city of Malabon and slowly spread, eventually affecting Valenzuela, Navotas, and Caloocan as well. Also, by looking at the third and fourth quarters of the year 2011, it can be observed that dengue incidence appeared to subside over time due to the low-high clusters of Caloocan and Marikina in the third quarter, and the low-low cluster of Quezon City in the fourth quarter.

The southern cities such as Las Piñas and Muntinlupa, all exhibited relatively low counts compared to the other cities, resulting in clusters of low rates computed by SaTScan. For all the four years 2010 to 2013, the cities of Las Piñas and Muntinupa were constantly regarded as clusters of low dengue incidence under either the SaTScan analysis. However, for the fourth quarter of years 2012 and 2013, Muntinlupa was identified as a lone cluster of high dengue incidence. Additionally, Las Piñas and Parañaque City are also identified as clusters of high dengue incidence in the fourth quarter of the year 2013. On the other hand, the northern cities, Malabon, Valenzuela, Caloocan, and Navotas were consistently identified as clusters of relatively high dengue incidence for the years 2010 and 2011. Malabon was constantly regarded as a region with high dengue incidence. The city was either included in a circle or was considered a lone cluster of high incidence for 15 out of the 16 quarters.

The relative risk was computed per city per quarter, according to the results of the Kulldorff's SaTScan statistic. In particular, for the first quarter of 2011, the cluster consisting of Valenzuela, Malabon, and Navotas exhibited a

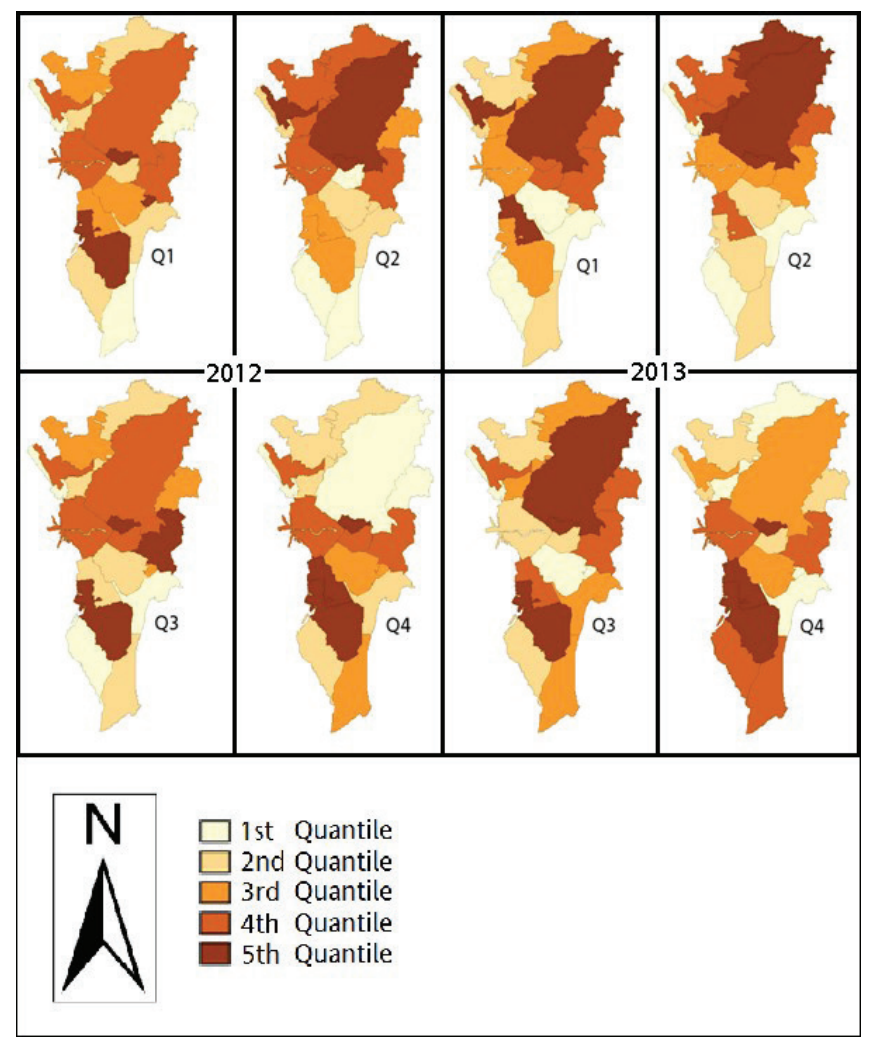

Figure 3. Quintile Maps of Quarterly Dengue Incidence Rates for years 2010 and 2011.

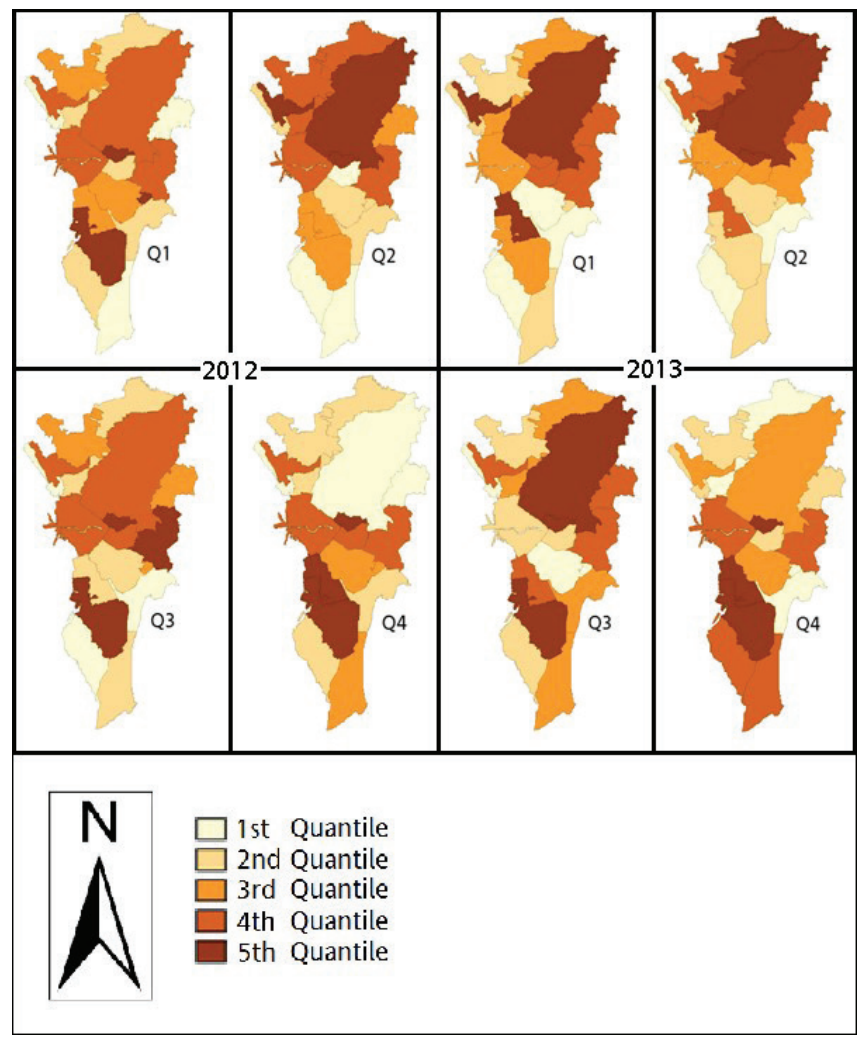

Figure 4. Quintile Maps of Quarterly Dengue Incidence Rates for years 2012 and 2013. 


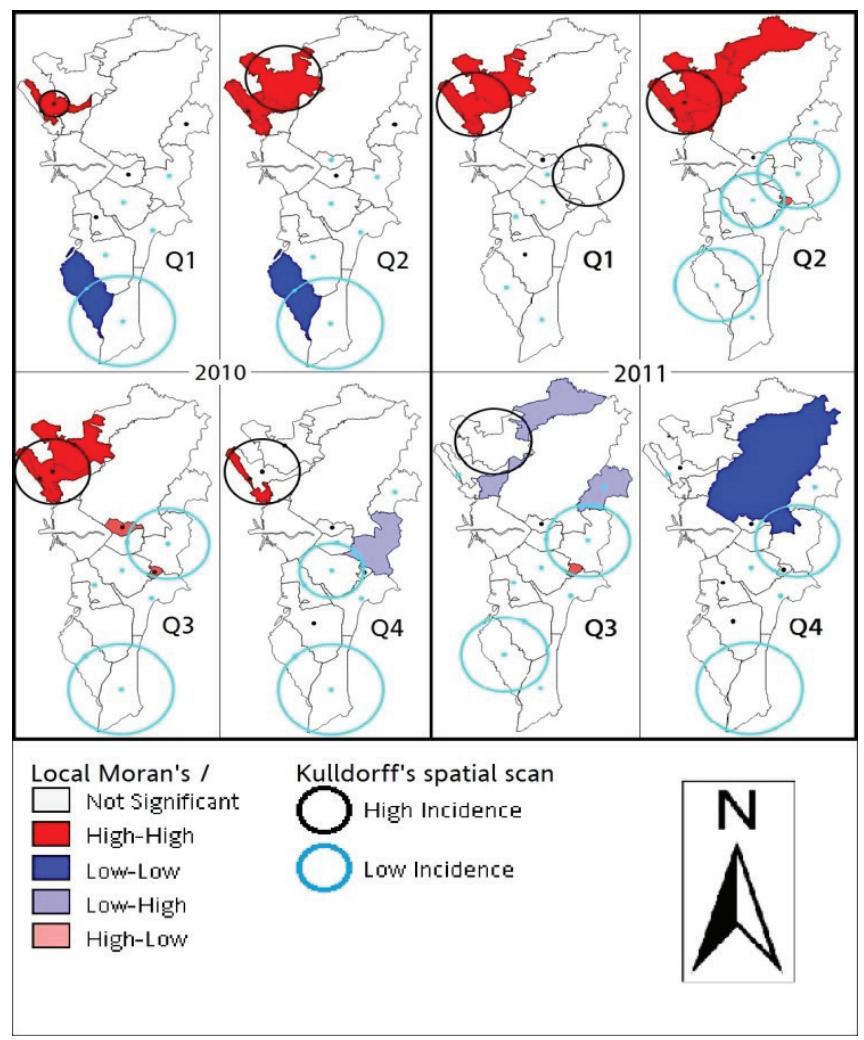

Figure 5. SaTScan-Generated Circles Overlain on Local Moran's I Cluster Maps for years 2010 and 2011.

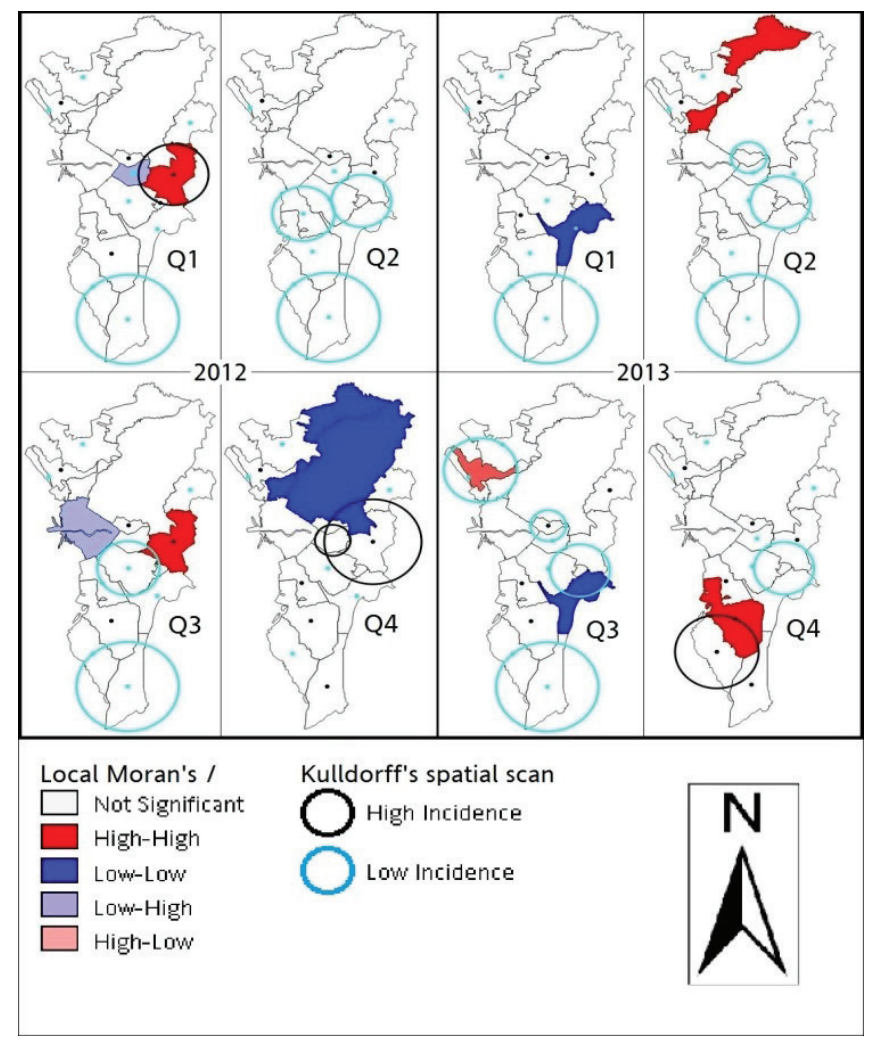

Figure 6. SaTScan-Generated Circles Overlain on Local Moran's I Cluster Maps for years 2012 and 2013.
$60 \%$ higher chance of contracting the disease compared to the other cities in NCR located outside the cluster. For 11 out of the 16 quarters, Malabon was identified as a statistically significant cluster of relatively high dengue incidence rates compared to the incidence rates outside the generated circles. Peak relative risk is observed in the sole cluster of high dengue incidence that occurred in Navotas during the second quarter of 2010. It was observed that during this quarter, incidence rates of dengue in Navotas were seven times higher than that of the incidence rates in other cities. The results show that most of the significant clusters of relatively high dengue incidence rates occur in the northern region of NCR. On the other hand, it was observed that in the second quarter of 2012, the cluster consisting of Las Piñas and Muntinlupa exhibited a 58\% lower chance of contracting disease in comparison to the other cities outside the cluster. For 14 out of the 16 quarters, Muntinlupa was identified as a cluster of relatively low dengue incidence rates. Las Piñas, however, was constantly regarded as a significant cluster of relatively low dengue incidence rates for 15 out of 16 quarters considered in the study. Peak relative risk in Las Piñas occurred at the fourth quarter of 2012, and was observed to be equal to 0.84 . This suggests that for these 15 quarters, Las Piñas consistently observed at least $16 \%$ lower dengue incidence rates compared to the other cities.

The results from both the Kulldorff's SaTScan statistics and the local Moran's I revealed similar patterns in the distribution of dengue incidence in NCR. However, some notable differences can be observed. Pateros was considered a cluster of relatively high dengue incidence under the local Moran's I analysis, while the Kulldorff's SaTScan statistics included this city in a cluster of low dengue incidence for the third quarter of 2010, and the second and third quarters of 2011. Also, for the third quarter of 2013, Malabon was included in a circle of low dengue incidence under Kulldorff's SaTScan statistics, but was identified as a highlow region under local Moran's I. Lastly, the Kulldorff's SaTScan statistics identified a significantly larger number of clusters compared to the local Moran's I. The results show that the northern cities i.e. Malabon, Navotas, Caloocan and Valenzuela are more prone to having clusters of high dengue incidence. This may be due to the fact that these cities are more susceptible to flooding when rainfall occurs, and in turn creates more habitats for which mosquitoes may breed in. Studies also show that these four cities have the highest poverty indices in $\mathrm{NCR}^{18}$, which seems to satisfy the notion that substandard housing and unplanned urbanization may be catalysts for vector breeding. ${ }^{5}$ Although Manila is the densest city in NCR in terms of population ${ }^{19}$, it did not register as a cluster of high dengue incidence for any quarter in the four years considered for the study. It is also observable that the southern cities (i.e., Las Piñas and Muntinlupa) are less likely to have high 
clusters of dengue incidence. It is worth noting that these cities are less susceptible to flooding, as verified by the Mines and Geosciences Bureau (MGB)'s flood map. ${ }^{20}$ At the same time, the poverty incidences for these cities are relatively low. ${ }^{18}$

\section{Conclusion}

The Moran's I analysis revealed that there was significant clustering in NCR. Further analyses using local Moran's I and Kulldorff's SaTScan statistics showed that the northern cities in NCR exhibited relatively high spatial autocorrelation due to high dengue incidence. Moreover, the overall results suggested that there was a movement of dengue incidence clustering over time. These analyses are vital in disease surveillance and prevention. Health organizations may be able to use these results to properly allocate their resources. Additionally, the presence of spatial autocorrelation in dengue incidence suggests possible measures of early detection for disease surveillance. Health experts from one city may be able to use the outbreak information in neighboring cities as an early warning device for a possible outbreak in their city.

The observed results showed that despite the presence of many factors in Manila and Mandaluyong that were believed to enhance the growth of dengue vectors and infection, these two cities were not identified as clusters of high dengue incidence. This may be due to vector management programs and anti-dengue initiatives being implemented in these cities, such as the Flood Control Bayanihan Zone Alliances, which focuses its efforts on areas within and around these cities. Thus, these cities may be used as models for dengue prevention programs in other cities.

\section{Acknowledgments}

The researchers would like to thank the Department of Health's Regional Epidemiology and Surveillance Unit for providing the data needed regarding reported dengue cases in NCR.

\section{Statement of Authorship}

All authors have approved the final version submitted.

\section{Author Disclosure}

All the authors declared no conflicts of interest.

\section{Funding Source}

None.

\section{References}

1. White K. Dengue fever. New York, NY: The Rosen Publishing Group Inc; 2004. pp. 5-6.

2. Paddock M. Medical News Today: What is dengue fever? What causes dengue fever? [Online]. 2010 [cited 2014 May]. Available from http://www.medicalnewstoday.com/articles/179471.php.

3. World Health Organization. WPRO Dengue Situation Updates [Online]. November 13, 2013 [cited 2014 May]. Available from http://www.wpro. who.int/emerging_diseases/DengueSituationUpdates/en/index.html.

4. Department of Health. National dengue prevention and control program [Online]. 2011 [cited 2014 May]. Available from http://www.doh. gov.ph/content/national-dengue-prevention-and-control-program.html.

5. World Health Organization. Global strategy for dengue prevention and control. Geneva, Switzerland [Online]. 2012 [cited 2014 May]. Available from http://www.who.int/immunization/sage/meetings/2013/april/5_ Dengue_SAGE_Apr2013_Global_Strategy.pdf.

6. World Health Organization. Dengue and severe dengue [Online]. 2013 [cited 2014 May]. Available from http://www.who.int/mediacentre/ factsheets/fs117/en/index.html.

7. Beattie P. The Burrill Report: The world's tropical zone - rich beauty, rare opportunity [Online]. 2010 [cited 2014 May]. Available from http://www.burrillreport.com/article-the_world\%E2\%80\%99s_tropical_ zone_\%E2\%80\%93_rich_beauty_rare_opportunity.html.

8. Shepard DS, Suaya JA, Halstead SB, et al. Cost-effectiveness of a pediatric dengue vaccine. Vaccine. 2004 Mar 12; 22(9-10):1275-80.

9. Centers for Disease Control and Prevention. Dengue and Climate [Online]. 2012 [cited 2014 May]. Available from http://www.cdc. gov/dengue/entomologyEcology/climate.html.

10. United Nations Population Fund. State of world population 2007. New York, New York; 2007. pp. 55-66.

11. Frialde M. The Philippine Star: MMDA launches bayanihan anti-flood project [Online]. January 11, 2011 [cited 2014 May]. Available from http://www.philstar.com/metro/649741/mmda-launches-bayahihan-antiflood-project.

12. Sugumaran R, Larson S, DeGroote JP. Spatio-temporal cluster analysis of county-based human West Nile virus incidence in the continental United States. Int J Health Geogr. 2009; 8:43.

13. Castillo K, Korbl B, Stewart A, Gonzalez J, Ponce F. Application of spatial analysis to the examination of dengue fever in Guayaquil, Ecuador. Procedia Environ Sci. 2011; 7:188-93.

14. World Health Organization. Dengue [Online]. 2014 [cited 2014 July]. Available from http://www.wpro.who.int/Philippines/areas/ communicable_diseases/dengue/en/.

15. Waller L, Gotway C. Applied spatial statistics for public health data. Hoboken, New Jersey:John Wiley \& Sons, Inc,; 2004. pp. 227-31.

16. Anselin L. Local indicators of spatial association. Geogr Anal. 1995; 27(2):93-115.

17. Kulldorff M. A spatial scan statistic. Commun Stat Theory Methods. 1997; 26(6):1481-96.

18. National Statistics Coordination Board. City and municipal-level small area poverty estimates [Online]. 2009 [cited 2014 July]. Available from http://www.nscb.gov.ph/poverty/2009_SAE/2009\%20FINAL\%20sae.pdf.

19. National Statistics Office. Special release: 2010 Census of population and housing - final results [Online]. 2012 [cited 2014 July]. Available from http://nso-ncr.ph/special\%20release/2010\%20CPH\%20Special\%20Release_ NCR.pdf.

20. Mines and Geosciences Bureau. [Interactive map illustrating flood prone areas in NCR]. Geohazard map of the Philippines: Flood susceptibility map and landslide susceptibility map [Online]. 2009 [cited 2014 July]. Available from http://www.mapcentral.ph/mgb/index.cfm/maptype= Flood $\&$ areatype $=$ municipality \&code $=997601000 \&$ listform_prov_code $=99$ 3900000 . 